\title{
goosecoid is not an essential component of the mouse gastrula organizer but is required for craniofacial and rib development
}

\author{
Jaime A. Rivera-Pérez', Moisés Mallo², Maureen Gendron-Maguire ${ }^{2}$, Thomas Gridley² \\ and Richard R. Behringer ${ }^{1, *}$ \\ ${ }^{1}$ Department of Molecular Genetics, The University of Texas M. D. Anderson Cancer Center, Houston, Texas 77030, USA \\ ${ }^{2}$ Roche Institute of Molecular Biology, Roche Research Center, Nutley, New Jersey 07110, USA \\ *Author for correspondence
}

\section{SUMMARY}

goosecoid (gsc) is an evolutionarily conserved homeobox gene expressed in the gastrula organizer region of a variety of vertebrate embryos, including zebrafish, Xenopus, chicken and mouse. To understand the role of gsc during mouse embryogenesis, we generated $g s c$-null mice by gene targeting in embryonic stem cells. Surprisingly, gsc-null embryos gastrulated and formed the primary body axes; gsc-null mice were born alive but died soon after birth with numerous craniofacial defects. In addition, rib fusions and sternum abnormalities were detected that varied depending upon the genetic background. Transplantation experiments suggest that the ovary does not provide gsc function to rescue gastrulation defects. These results demonstrate that gsc is not essential for organizer activity in the mouse but is required later during embryogenesis for craniofacial and rib cage development.

Key words: gastrulation, skeleton, craniofacial development, goosecoid, mouse

\section{INTRODUCTION}

The classic embryological experiments of Spemann and Mangold (1924) lead to the development of the concept of the organizer. They showed that, when the dorsal blastopore lip from a gastrula stage amphibian embryo was transplanted to the presumptive ventral side of another comparably staged embryo, a secondary axis developed. Their ability to follow the fates of the grafted tissue demonstrated that the donor tissue was able to change the cell fates of the surrounding host cells to participate in the development of the secondary axis. Candidate genes that may participate in this complex embryological phenomenon have been identified. A subset of these genes encode transcription factors, perhaps the most notable among these is $g s c$.

gsc is a homeobox-containing gene that was originally isolated in Xenopus from a dorsal blastopore lip cDNA library (Blumberg et al., 1991). During embryogenesis, gsc is expressed before the initiation of gastrulation in the dorsal marginal zone of the Xenopus embryo above the dorsal lip (Cho et al., 1991). gsc transcription can be activated by the mesoderm inducer activin (Cho et al., 1991; Green et al., 1992; Steinbeisser et al., 1993), suggesting a role for $g s c$ in mesoderm induction. In addition, injection of $g s c$ mRNA into Xenopus embryos can induce the formation of a secondary body axis, demonstrating that gsc can initiate organizer activity (Cho et al., 1991). These observations suggest that gsc may be an essential component of the vertebrate gastrula organizer. gsc homologs have also been isolated in other vertebrate species, including zebrafish, chick and mouse (Stachel et al., 1993; Shulte-Merker et al., 1994; Izpisúa-Belmonte et al., 1993; Blum et al., 1992). In zebrafish, $g s c$ is expressed in the anterior region of the embryonic shield and axial hypoblast, and later during embryogenesis in larval cranial neural crest derivatives (Stachel et al., 1993; Shulte-Merker et al., 1994). Lithium treatment of zebrafish embryos results in radialized embryos that are hyperdorsalized (Stachel et al., 1993). gsc expression in these lithium-treated embryos is elevated and radialized. These results suggest that lithium treatment induces ectopic $g s c$ expression that leads to the generation of multiple organizer fields. In the chick, gsc-expressing cells are first detectable in Koller's sickle, a group of cells near the posterior margin zone of the unincubated egg that, when transplanted into another embryo, can induce a secondary axis (Izpisúa-Belmonte et al., 1993). Thus, studies in zebrafish and chick also implicate $g s c$ as a factor with organizer activity.

In the mouse, $g s c$-expressing cells are found transiently at the anterior end of the primitive streak of the gastrula between E6.4 and E6.8 (Blum et al., 1992). Transplantation studies into Xenopus or mouse embryos have demonstrated that this region of the mouse gastrula possesses some of the organizer functions of the dorsal blastopore lip (Blum et al., 1992; Beddington, 1994). gsc expression reappears at E10.5 (Gaunt et al., 1993). At these later stages of embryogenesis, gsc transcripts are found in undifferentiated tissues and persist as those tissues undergo morphogenesis (Gaunt et al., 1993). Between E10.5 
and E14.5, gsc transcripts are detected in the lower jaw and the tongue, the eustachian tube and base of the auditory meatus, the mesenchyme surrounding the nasal pits that form the nasal chambers, and the proximal limb buds and the vetrolateral body wall that form the proximal limb structures and ventral ribs. These findings suggest that, in addition to a potential role in gastrulation, $g s c$ may also be required later during mouse embryogenesis for craniofacial, limb and thoracic development.

To determine the requirement of $g s c$ during mouse development, we generated gsc-null mice by gene targeting in mouse embryonic stem (ES) cells. Gastrulation progressed normally in $g s c$-null embryos and all of the primary body axes formed correctly; gsc-null mice were born alive but died soon after birth with craniofacial defects. In addition, rib fusions and sternum abnormalities were detected. These results demonstrate that $g s c$ is not an essential component of the gastrula organizer in the mouse but is required later during embryogenesis for craniofacial and rib cage development.

\section{MATERIALS AND METHODS}

\section{Deletion of the gsc gene in mouse ES cells}

A $129 / \mathrm{SvEv}$ mouse genomic library (Stratagene) was screened with a probe containing nucleotides 1837 to 2166 of the mouse gsc genomic sequences (GenBank accession number M85271; Blum et al., 1992). The probe is a subfragment of the $g s c$ locus generated by PCR amplification of mouse genomic DNA. One phage clone that hybridized with the probe was subcloned into pBluescript, and its $g s c$ identity was verified by DNA sequencing. A $2.9 \mathrm{~kb}$ Asp718-SalI upstream fragment and a $2.0 \mathrm{~kb}$ downstream region from the NotI$X b a \mathrm{I}$ sites, were used to construct a replacement vector (Fig. 1A). A PGKneobpA neomycin resistance expression cassette (Soriano et al., 1991) was inserted in either forward or reverse orientation relative to the direction of $g s c$ transcription between the two $g s c$ regions. A MC1tkpA herpes simplex virus thymidine kinase expression cassette was added onto the $3^{\prime}$ arm of homology to enrich for homologous recombinants using negative selection with 1-(2-deoxy-2-fluoro- $\beta$-Darabinofuranosyl)-s-iodouracil (FIAU) (Mansour et al., 1988). The targeting vectors can be linearized at unique Asp718 sites outside of the homology. $10 \mu \mathrm{g}$ of linearized targeting vector was electroporated into $10^{7} \mathrm{AB}-1 \mathrm{ES}$ cells that were subsequently cultured in the presence of G418 and FIAU (Soriano et al., 1991; McMahon and Bradley, 1990). 154 G418/FIAU-resistant ES clones were initially screened by HindIII digestion and hybridization with a unique $5^{\prime}$ probe external to the region of vector homology. Correctly targeted clones were then expanded for further Southern blot analysis by EcoRI/EcoRV digestion and hybridization with a unique $3^{\prime}$ probe external to the vector homology. 39 correctly targeted ES clones were identified. The overall targeting frequency for both vectors from two independent electroporations was approximately $1 / 4$ G418/FIAU-resistant colonies screened.

\section{Generation of chimeric mice and germline transmission of the gsc deletion alleles}

Four of the gsc mutant ES clones were microinjected into B6-albino blastocysts and the resulting chimeric embryos were transferred to the uterine horns of day 2.5 pseudopregnant foster mothers (Bradley, 1987). Chimeras were identified among the resulting progeny by their pigmented fur (ES-derived) and were subsequently bred with B6albino mates. Three of the mutant ES clones (one with neo in the forward orientation and two with neo in the reverse orientation) were found to be capable of contributing to the germline of chimeric mice.
A
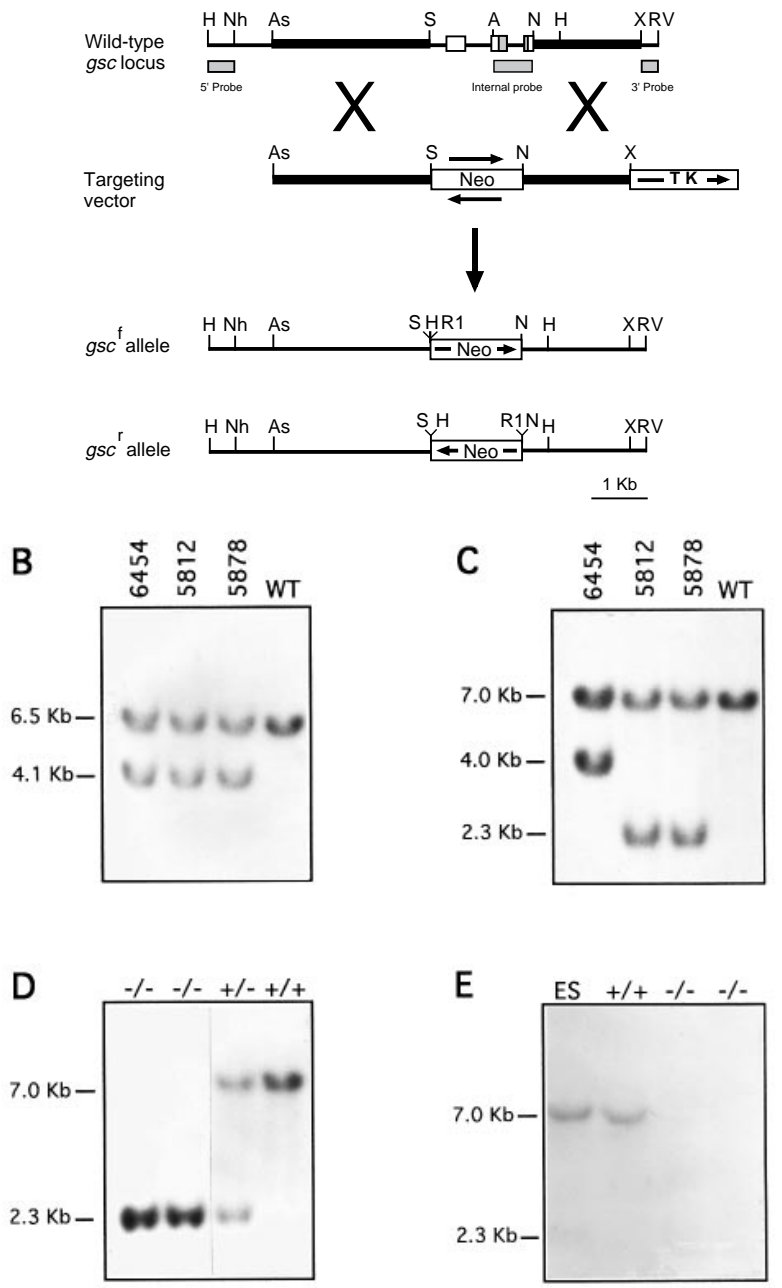

Fig. 1. Targeted deletion of the $g s c$ gene in ES cells and mice. (A) Strategy for targeted deletion of the $g s c$ gene. $g s c$ is encoded by three exons contained within a 1.9-kb SalI-NotI DNA fragment; the homeobox (shaded) is split between the second and third exons. The targeting vector possesses a total of $4.9 \mathrm{~kb}$ of homologous sequence including a $2.9 \mathrm{~kb}$ upstream region as a $5^{\prime}$ arm of homology and a 2.0 $\mathrm{kb}$ downstream region as a $3^{\prime}$ arm isolated from a mouse strain 129/Sv genomic library (thick closed bars). A PGK-neo cassette (Soriano et al., 1991) (neo) was inserted between the two arms of homology in either forward $\left(g s c^{\mathrm{f}}\right)$ or reverse $\left(g s c^{\mathrm{r}}\right)$ orientation relative to the direction of $g s c$ transcription. The neo cassette replaces the $g s c$-containing SalI-NotI DNA fragment and introduces novel restriction sites for Southern analysis. A MC1-tk cassette (TK) was added for negative selection (Mansour et al., 1988). Unique Southern probes were identified that lie $5^{\prime}$ and $3^{\prime}$ outside of the vector homology. $(\mathrm{B}, \mathrm{C})$ Southern blot analysis of DNA from $g s c^{\mathrm{f}}$ mutant ES clone 6454, $g s c^{\mathrm{r}}$ mutant ES clones 5812 and 5878, and wild-type (WT) ES cells: HindIII digest hybridized with the $5^{\prime}$ probe (B); EcoRI/EcoRV digest hybridized with the 3' probe (C). (D) Southern blot analysis of tail DNA from the offspring of heterozygote matings from the 5878 line. EcoRI/EcoRV digest hybridized with the $3^{\prime}$ probe. (E) Southern blot analysis of DNA from wild-type and homozygous mutant mice from the 5812 line. EcoRI/EcoRV digest hybridized with a $g s c$ probe contained within the deletion. A, ApaI; As, Asp 718; H, HindIII; N, NotI; Nh, NheI; RI, EcoRI; RV, EcoRV; S, SalI; X, XbaI. +/+, wild-type; +/-, heterozygous; -I-, mutant. 
Table 1. Genotype of newborn offspring derived from heterozygous gsc parents

\begin{tabular}{lccccc}
\hline Allele/Clone & Litters & Wild-type & Heterozygous & Mutant & Total \\
\hline$g s c^{r}(5878)$ & 15 & $23(19.5 \%)$ & $69(58.5 \%)$ & $26(22 \%)$ & 118 \\
$g s c^{r}(5812)$ & 7 & $11(30 \%)$ & $16(43 \%)$ & $10(27 \%)$ & 37 \\
$g s c^{f}(6454)$ & 11 & $12(17 \%)$ & $47(67 \%)$ & $11(16 \%)$ & 70 \\
\hline
\end{tabular}

Tail DNA from the pigmented pups that resulted from those matings was analyzed by Southern blotting with either of the probes used to identify $g s c$ heterozygotes. Chimeras were also bred with $129 / \mathrm{SvEv}$ females to establish the $g s c$ deletion alleles on the $129 / \mathrm{SvEv}$ inbred genetic background. Mice carrying the $g s c$ mutation were also backcrossed to B6 mice to initiate the generation of a congenic mouse line. gsc heterozygotes at generation 4 or 5 possess an inbreeding coefficient of 0.938 and 0.969 , respectively, and were used in this study.

\section{Ovary transplants}

gsc heterozygotes $(\mathrm{B} 6 \times 129$ hybrid genetic background) were interbred to establish timed matings. On E18.5, the pups were delivered by Ceasarian section. gsc-null pups were identified by their abnormal breathing behavior and, upon dissection, by air in the gut. Subsequent Southern blot analysis confirmed that they were $g s c$-null. Both ovaries from a $g s c$-null female were transplanted into the bursal sac of a B6×129 $F_{1}$ hybrid female approximately 3 weeks of age whose ovary had been surgically removed. The oviduct from the other uterine horn was surgically ablated leaving the endogenous ovary intact. After three weeks, the transplanted females were bred with gsc heterozygous males. In this scheme, the only way that gsc homozygous mutant progeny can be obtained is if the $g s c$-null ovary transplant was successful.

\section{Skeleton preparations}

Neonates were killed, skinned, eviscerated and fixed in $95 \%$ ethanol. Their skeletons were subsequently prepared by alkaline digestion and stained with alizarin red $\mathrm{S}$ for ossified bone and alcian blue 8GX for cartilage (Kochhar, 1973). For fetal cartilaginous skeletons, embryos were fixed in Bouin's fixative, washed, stained with alcian blue 8GX, dehydrated and cleared in 2:1 benzyl benzoate:benzyl alcohol (Jegalian and De Robertis, 1992).

\section{Histological analysis}

Embryos at E18.5 and E15.5 (not shown) were fixed in Bouin's solution, dehydrated in graded alcohols and embedded in paraffin. $10 \mu \mathrm{m}$ sections were cut and stained with haematoxylin and eosin.
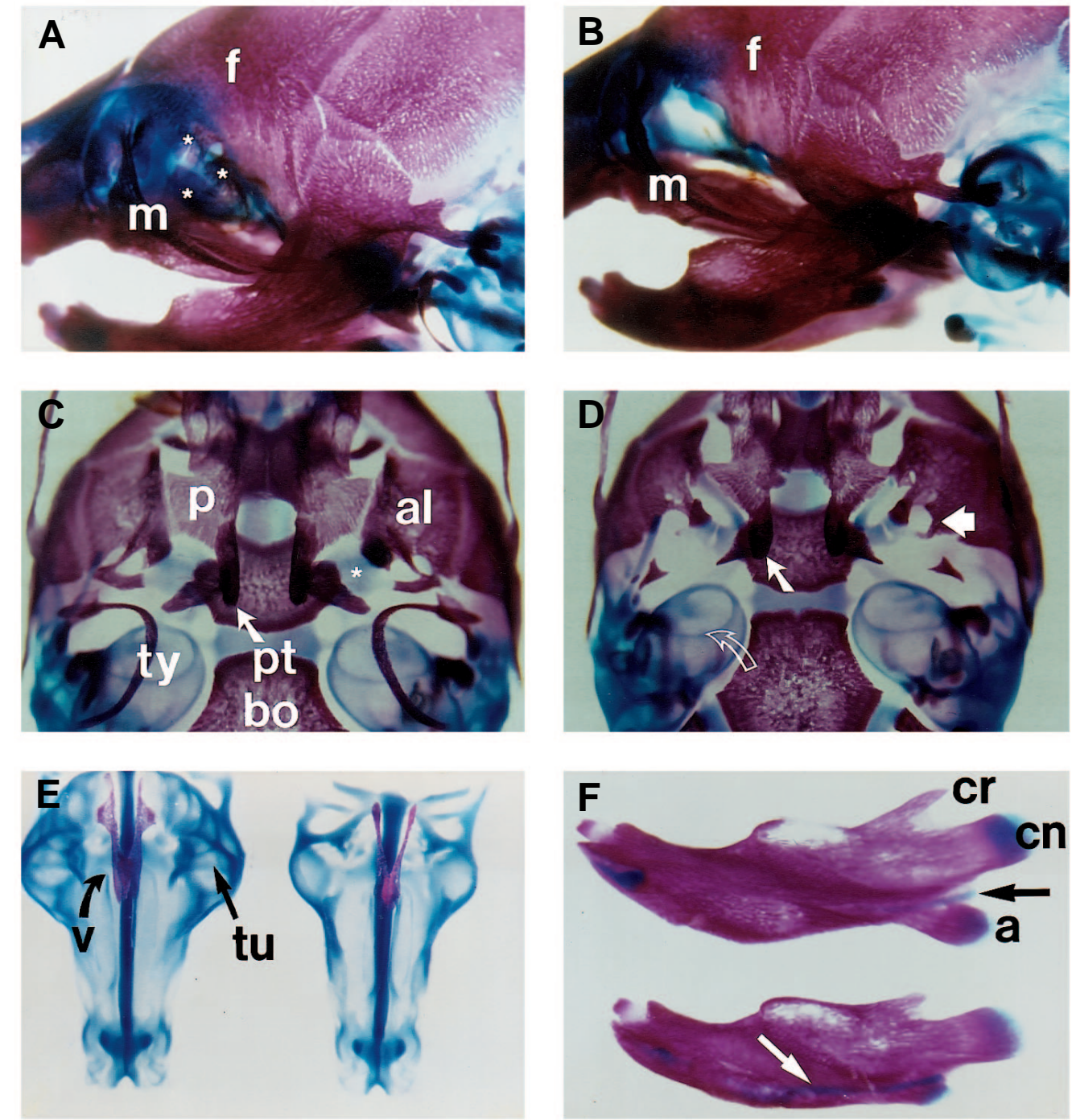

Fig. 2. Craniofacial abnormalities of $g s c$-null mice. Skeleton preparations of wild-type neonates (A,C) and $g s c$-null littermates (B,D) (A,B) Lateral view of skull. $g s c$-null mice have a reduction of the orbital processess (asterisks) of the maxillary (m) and frontal (f) bones. (C,D) Ventral view of skull with jaw removed (the nasal region is at the top). Portions of several bones are reduced or malformed in the mutants. The palatine bone (p) has reduced wings and palatine shelves. The alisphenoid bone (al) has a malformed area of foramens (short solid arrow) and the cartilage that unites it with the basisphenoid bone is split in two (asterisk). The pterygoid bone (pt) is reduced in size (white slanted arrow) and the tympanic ring bone (ty) is absent in the mutants (open arrow). (E) Ventral view of dissected nasal cartilage. Wildtype (left) and $g s c$-null (right). The anlagen of the turbinal bones (tu) are absent in the $g s c$ mutant. The vomer bone (v) is reduced in the mutant. (F) Medial view of the right mandible of wild-type (top) and $g s c$ null (bottom) mice. The mandible of the gsc-null mouse is shorter. In $g s c$ mutants, the coronoid (cr) and angular (a) processes are reduced in size, whereas the condilar ( $\mathrm{cn}$ ) process is normal. A groove extending along the mandible is observed in the $g s c$-null mice, so that the Meckel's cartilage (white arrow) is now visible on the inner medial surface of the jaw, which is not seen in the control (black arrow). 


\section{RESULTS}

\section{Generation of two gsc-null alleles in the mouse germline}

The mouse gsc gene is encoded by three exons (Blum et al., 1992). To mutate the $g s c$ gene in mouse embryonic stem (ES) cells, we generated two different targeting vectors, both of which delete the entire GSC protein-coding region (Fig. 1A). The targeting vectors differed only with respect to the orientation of the neomycin (neo) selectable marker relative to the direction of $g s c$ transcription. The allele generated by the vector with the neo marker in the forward orientation was designated $g s c^{\mathrm{f}}$ and the allele generated by the vector with the neo marker in the reverse orientation was designated $g s c^{\mathrm{r}}$. When these vectors are homologously recombined with the mouse genome, novel restriction enzyme sites are introduced (Fig. 1A). Correctly targeted clones for both $g s c^{\mathrm{f}}$ and $g s c^{\mathrm{r}}$ can therefore be detected by the presence of an additional $4.1 \mathrm{~kb}$ mutant band when digested with HindIII and hybridized with a $5^{\prime}$ probe external to the region of vector homology or for $g s c^{\mathrm{f}}$ by the presence of a $4.0 \mathrm{~kb}$ mutant band and for $g s c^{\mathrm{r}}$ by the presence of a $2.3 \mathrm{~kb}$ mutant band when digested with EcoRI and EcoRV and hybridized with a $3^{\prime}$ probe external to the region of vector homology (Fig. 1B). Correct targeting with either vector results in the deletion of the entire GSC protein coding region, thereby creating null alleles. Correctly targeted ES clones were obtained for both vectors at a frequency of approximately $1 / 4$ G418/FIAU resistant colonies screened. Three correctly targeted ES clones (one for $g s c^{\mathrm{f}}$ and two for $g s c^{\mathrm{r}}$ ) successfully contributed to the germline of chimeric mice generated by blastocyst injection (Fig. 1B,C). The phenotype of $g s c$-null mice from these three independently derived ES clones were identical. In addition, the phenotype of $g s c^{\mathrm{f}} / g s c^{\mathrm{r}}$ mice was also identical to each of the $g s c^{\mathrm{f}}$ and $g s c^{\mathrm{r}}$ homozygous mutants.

\section{gsc-null mice are born alive without axial patterning defects}

Mice heterozygous for the gsc deletion alleles appeared normal and were fertile. Mice homozygous for either of the two $g s c$ mutant alleles were recovered alive at birth (Fig. 1D) and were overtly indistinguishable from their wild-type or heterozygous littermates. The genotypes of the offspring from heterozygote crosses followed predicted Mendelian frequencies, suggesting that homozygous mutant mice were not being lost during embryonic development (Table 1). Southern blot analysis using gsc coding sequences as a probe confirmed that $g s c$ homozygous mutant mice did not contain $g s c$ coding sequences, demonstrating that a null allele had been generated (Fig. 1E).

The recovery of $g s c$-null mice at birth without axial defects suggested that the patterning events that take place during gastrulation had occurred correctly without gsc function. Although gastrulation had clearly occurred, it was still possible that the absence of $g s c$ could result in a delay of early embryogenesis and that later in development $g s c$-null embryos could catch up with their wild-type and heterozygous littermates. However, at E7.5, gsc-null embryos had normal morphology and expressed HNF-3 $\beta$ protein (Sasaki and Hogan, 1993; Ang et al., 1993) correctly in the anterior midline and the node (not shown), a structure that possesses a subset of the functions of the Spemann organizer (Beddington, 1994). These results suggest that $g s c$ null embryos are able to gastrulate and organize early embryonic pattern with correct developmental timing. Therefore, in the mouse $g s c$ is not required for either mesoderm or axis formation.

\section{gsc-null mice are born from females with gsc-null transplanted ovaries}

In zebrafish and Xenopus, gsc transcripts are detected in oocytes (De Robertis et al., 1992; Stachel et al., 1993; Schulte-Merker et al., 1994), suggesting that maternal stores of $g s c$ RNA or protein may play a role in early embryonic patterning. Thus, the recovery of $g s c$-null mice at birth from heterozygous mothers could be due to the rescue of the mutants during gastrulation by a maternal source of $g s c$ function. It is currently unknown whether gsc RNA or protein is present in the mouse oocyte, and the lack of an antibody to GSC protein precludes a judgement about a maternal source of GSC protein. To address the question of a maternal source of $g s c$ function directly, we transferred the ovaries from $g s c$-null pups recovered by Ceasarian section at E18.5 into histocompatible $g s c$-wild-type recipient females that had been rendered incapable of producing wild-type oocytes. In this way, the host females would produce oocytes from the $g s c$-null ovaries that would lack gsc transcripts and protein. The females carrying the transplanted $g s c$-null ovaries were subsequently bred with $g_{s c}$ heterozygous males. Six pups were born alive from two of these females and four of these pups (three from one female and one from the other) were indistinguishable in phenotype from $\mathrm{gsc}^{-}$ null mice born from heterozygous matings. Genotyping by Southern analysis confirmed that these four pups were gsc homozygous mutants; the other two pups were heterozygotes. These results suggest that ovarian tissues do not provide $g s c$ function to rescue gastrulation defects in $g s c$-null mice.

\section{Neonatal lethality with craniofacial and rib cage abnormalities in gsc-null mice}

$g s c$-null mice never fed and all died within 24 hours after birth. The mutants could not suckle when physically placed upon the mother's nipples but could accumulate milk in their stomachs when forcefed, suggesting that the pathway from the mouth to the stomach was intact. In addition, the mutants had difficulty breathing which was associated with air in the stomach and intestines, and a pale body color.

Skeletal analysis of $g s c$-null neonates revealed numerous craniofacial and rib cage abnormalities. In the skull, the orbital processes of the maxillary and frontal bones that support the eye were reduced (Fig. 2A,B). In addition, the tympanic ring bone, which normally supports the tympanic membrane (eardrum), was absent (Fig. 2C,D). Within the middle ear, the manubrium and processus brevis of the malleus were smaller, whereas the incus and stapes were normal (not shown). In addition, several bones at the base of the skull were malformed, including the palatine, maxillary, alisphenoid and pterygoid bones (Fig. 2A-D). There were also significant alterations in the nasal region, including the lack of the anlagen for the turbinal bones that form the chambers of the nasal cavity (Fig. 2E). Furthermore, the mandible was shortened and, although the condilar process was normal, the coronoid and angular processes were diminished (Fig. 2F). A groove, extending along Meckel's cartilage of the mandible, was observed in the mutants but was not found in controls. The variations in mandible development were already apparent at E13.5. The craniofacial abnormalitites were detected in all 62 of the $g s c$-null mice analyzed. Although $g s c$ is abundantly expressed in the developing limbs, 
Table 2. Genetic background and phenotypic variation in gsc null mice

\begin{tabular}{llccc}
\hline $\begin{array}{l}\text { Genetic } \\
\text { background }\end{array}$ & Allele/clone & $\begin{array}{c}\text { Craniofacial } \\
\text { defects* }\end{array}$ & $\begin{array}{c}\text { Rib cage } \\
\text { defects }\end{array}$ & $\begin{array}{c}\text { Total } \\
\text { analyzed }\end{array}$ \\
\hline $\begin{array}{l}\text { F } 2 \text { Hybrid } \\
\text { C57BL/6×129SvEv }\end{array}$ & $g s c^{r}(5878)$ & $35(100 \%)$ & $19(54 \%)$ & 35 \\
$\begin{array}{l}\text { Coisogenic } \\
\text { 129SvEv }\end{array}$ & $g s c^{r}(5878)$ & $20(100 \%)$ & $3(15 \%)$ & 20 \\
$\begin{array}{c}\text { Congenic } \\
\text { C57BL/6 }\end{array}$ & $g s c^{r}(5878)$ & $20(100 \%)$ & $14(70 \%)$ & 20 \\
\end{tabular}

*Analysis for all bones affected by the mutation as described in text.

no skeletal abnormalities were detected in the limbs of any of the $g s c$-null mice.

Additional craniofacial defects were noted upon histological

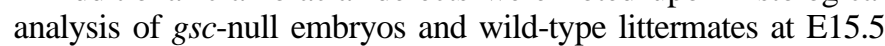
and E18.5. As observed in the skeletal preparations, gsc-null mutant embryos lacked the anlagen of the turbinal bones and the ventrolateral walls of the nasal cavity (Fig. 3A,B). In addition, the glandular mucous epithelium that normally covers the nasal sinuses was mostly absent in the mutants. However, midline nasal structures, such as the nasal septum and the vomeronasal organs and cartilages, were present in the mutants, although the nasal septum did not fuse with the palate. In addition, middle ear development in the gsc-null embryos was abnormal. Although the tubotympanic recess had formed and was present adjacent to the otic capsule, the external acoustic meatus had not migrated very far internally. Therefore, the tympanic membrane, which is formed by the apposition of the tubotympanic recess and the external acoustic meatus, did not form in gsc-null mice (Fig. 3C,D). In the tongue, the genioglossus muscle showed aberrant insertions on Meckel's cartilage, rather than inserting on the symphysis of the mandible as in controls (Fig. 3E-H). In addition, the density of muscle fibers of the extrinsic muscles of the tongue was reduced in the mutants (Fig. 3G,H). The thyroid and thymus glands, which express $g s c$ during embryogenesis, were present in the mutants.

Rib fusions were detected in about $35 \%$ of the $62 \mathrm{gsc}$-null skeletons analyzed (Fig. 4A-D). The fusions occurred between the costal cartilages of the first and second ribs, although in one case the fourth and fifth ribs had fused. The rib fusions were unilateral, either on the left or right side, or bilateral. An additional $20 \%$ of the $g s c$-null skeletons had a different defect in rib cage development. In these skeletons, rather than rib fusions, a reduced number of ribs were attached to the sternum in comparison to controls. As in the case of the rib fusions, this variation in rib attachment was unilateral, on either side, or bilateral. Typically, the skeletons with rib fusions or abnormal numbers of attached ribs had sternum abnormalities characterized by modifications in sternebrae ossification, probably the result of incorrect rib attachment. The rib fusions were evident in mutant embryos at E14.0 (Fig. 4E).

The initial skeletal analyses were performed on a C57BL/6 (B6) $\times 129 \mathrm{~F}_{2}$ hybrid genetic background. We also examined the $g s c$ mutation on a $129 / \mathrm{SvEv}$ inbred genetic background and on a genetic background that was theoretically $>90 \%$ B6 (Table 2). All $g s c$-null 129 inbred mice or B6 mice died soon after birth with essentially the same craniofacial syndrome exhibited by

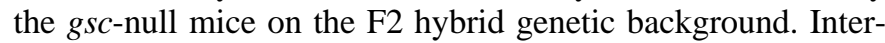
estingly, whereas the penetrance of the craniofacial abnormalities of the $g s c$-null mice were the same on both the 129 and B6 genetic backgrounds, the penetrance of the rib cage abnor- malities were different on these two genetic backgrounds. Rib fusions or changes in the normal number of ribs contacting the sternum were recorded as deviations of the normal pattern of rib cage development. Whereas approximately $55 \%$ of the $g s c$ null mice on the $\mathrm{F}_{2}$ hybrid background had rib cage abnormalities, only about $15 \%$ of the $g s c$-null mice had such defects on the 129 inbred background. In contrast, $70 \%$ of the $g s c$-null mice had rib cages defects on the B6 background. These results suggest that there is genetic variation between strains 129 and B6 that can suppress or enhance the frequency of rib cage defects caused by the $g s c$ mutation, respectively.

\section{DISCUSSION}

\section{gsc and the vertebrate gastrula organizer}

Previous studies had suggested that $g s c$ was an essential component of the vertebrate gastrula organizer (Cho et al., 1991; Blum et al., 1992; Izpisúa-Belmonte et al., 1993). These expectations were based upon the observations that $g s c$ was expressed in the organizer regions of four vertebrate species and that gain-of-function assays in Xenopus resulted in the development of secondary axes (Cho et al., 1991, Blum et al., 1992; Izpisúa-Belmonte et al., 1993). However, even in the gain-offunction assays, $g s c$ only had weak organizer activity; trunk duplications were most frequently induced and rarely were complete axes with head structures formed (Cho et al., 1991). In this study, we have determined the requirement of $g s c$ during mouse embryogenesis by generating a loss-of-function mutation in mice by gene targeting in ES cells. Our studies unequivocally demonstrate that embryonic expression of $g s c$ is not required for mesoderm induction or axis formation in mice.

In zebrafish and Xenopus, gsc is a maternally expressed transcript (De Robertis et al., 1992; Stachel et al., 1993; SchulteMerker et al., 1994). Thus, maternally derived gsc RNA or protein could be used by the early embryo for axial development. However, if there were a maternal gsc component in the mouse, it is unlikely that it would persist long enough (to E6.5 when gastrulation is initiated) in the developing embryo to be biologically relevant. Moreover, the recovery of $g s c$-null pups from a mating between a female mouse carrying $g s c$-null ovaries suggests that the ovary does not provide $g s c$ activity to the embryo for mesoderm formation or axial patterning. However, it is still formally possible that maternal contributions of GSC function exclusive of the ovary could effect a rescue.

One likely explanation for our results is that $g s c$ serves a redundant role with respect to the mouse gastrula organizer. Thus, other genes may exist in mice that provide organizer activity. Candidates for such genes include $H N F 3 \beta$ and Lim 1 because mutations in these genes lead to axial defects in mice 


\section{J. A. Rivera-Pérez and others}
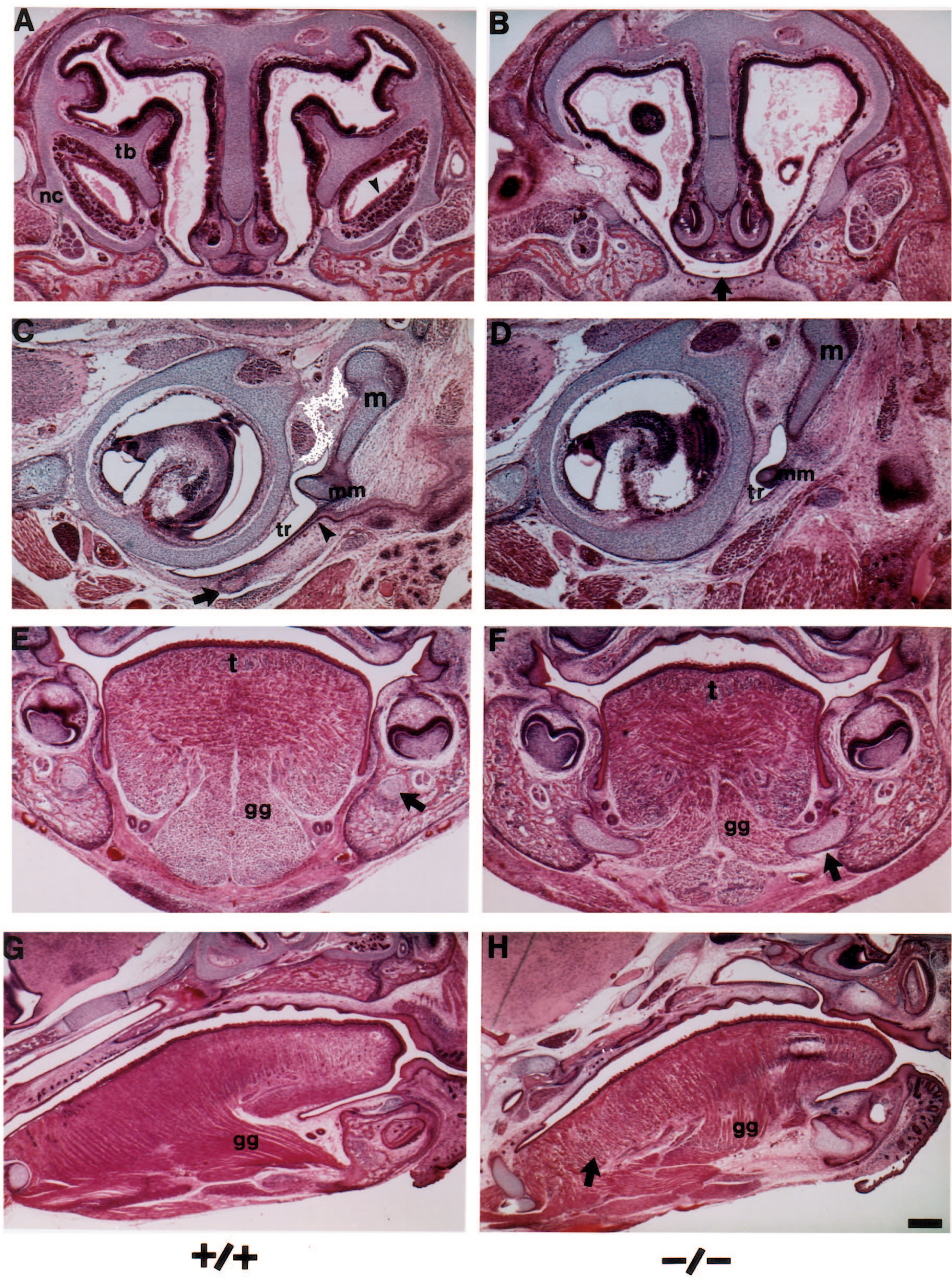
Fig. 3. Histological analysis of $g s c$-null mice. Wild-type (+/+) embryos (A,C,E,G) and $g s c$-null (-/-) littermates (B,D,F,H) were isolated at E18.5 and were sectioned in frontal (A-F) and sagittal $(\mathrm{G}, \mathrm{H})$ planes. $(\mathrm{A}, \mathrm{B})$ Mutant embryos exhibited multiple defects in the nasal region, including loss of the anlagen of the turbinal bones (tb) and the ventrolateral walls of the nasal capsule (nc), loss of the glandular mucous epithelium (arrowhead) and lack of fusion of the nasal septum to the palate (arrow). (C,D) In mutant embryos, the tympanic ring (arrow) was absent. The external acoustic meatus (arrowhead) did not extend into the region surrounding the otic capsule; thus, the tympanic membrane, which is formed by the apposition of the tubotympanic recess (tr) and the external acoustic meatus, did not form in the mutants. The manubrium (mm) of the malleus (m) of the mutants was smaller than controls. (E,F) In the mandible of wild-type embryos, Meckel's cartilage (arrow) is completely enveloped by the ossifying dentary bone. In mutants, Meckel's cartilage is not completely enveloped by the dentary bone and the genioglossus muscle (gg) of the tongue $(\mathrm{t})$ aberrantly inserts on Meckel's cartilage. $(\mathrm{G}, \mathrm{H})$ A sagittal section of the mutant embryo displays both the aberrant insertions of the genioglossus muscle (gg) and the decreased density of extrinsic muscle fibers (arrow) of the tongue. Abbreviations: gg, genioglossus muscle; m, malleus; mm, manubrium of the malleus; nc, nasal capsule; $t$, tongue; tb, turbinal bone; tr, tubotympanic recess. Scale bar: A,B,E,F, 160 $\mu$ m; C,D, $200 \mu \mathrm{m} ; \mathrm{G}, \mathrm{H}, 100 \mu \mathrm{m}$.
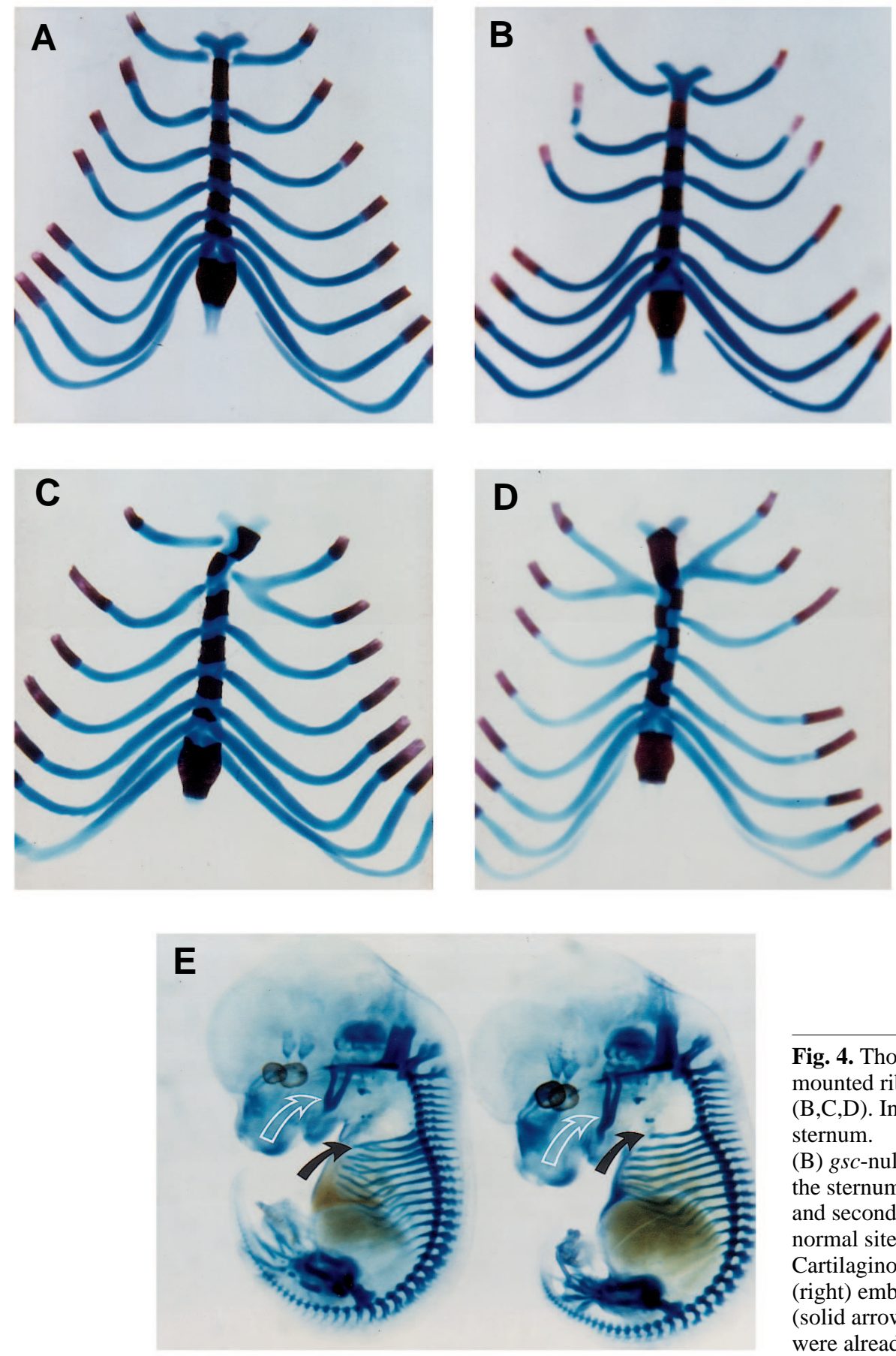

(Ang and Rossant, 1994; Weinstein et al., 1994; Shawlot and Behringer, 1995). It will be interesting to generate compound mutants with $g s c$ and these genes to reveal a required function for $g s c$ in organizer activity.

It is clear that ectopic high level expression of $g s c$ can dramatically alter the axial organization of the Xenopus embryo (Cho et al., 1991). In addition, elevated levels of radialized $g s c$ expression after lithium treatment correlates with the development of dorsalized zebrafish embryos (Stachel et al., 1993). These observations suggest that $g s c$ expression must be restricted both spatially and quantitatively for correct axis formation. It seems reasonable to suggest that it is critical for vertebrate embryos to maintain precise levels of organizer activity. A redundant or compensatory role for $g s c$ could be envisioned in which gsc expression would be modulated, probably by growth factors (Cho et al., 1991; Green et al., 1992; Steinbeisser et al., 1993), to compensate for variations in organizer activity levels to maintain them within a narrow window of action. This would provide a certain amount of flexibility in response to inductive signals or other environmental cues to maintain axial patterning in the embryo. Whatever the case may be, the results presented here provide important information required for the interpretation of abnormalities in germ layer and axis

Fig. 4. Thoracic skeletal abnormalities of $g s c$-null mice. Flat mounted rib cages from wild-type (A) and $g s c$-null mice (B,C,D). In wild-type mice, seven pairs of ribs contact the sternum.

(B) $g s c$-null rib cage with only six pairs of ribs attached to the sternum. (C,D) Rib fusions occurred between the first and second ribs and contacted the sternum close to or at the normal site of attachment for the second rib. (E)

Cartilaginous skeletons of E14.0 wild-type (left) and mutant (right) embryos with the forelimbs removed. Rib fusions (solid arrow) and mandible abnormalities (open arrows) were already evident at this stage. 
formation in vertebrate embryos with alterations in $g s c$ expression patterns.

\section{gsc and craniofacial and rib cage development}

The non-viability of $g s c$-null mice clearly demonstrates that there are required functions for $g s c$ during development. This essential role for $g s c$ is in craniofacial and rib cage morphogenesis. The craniofacial defects observed in the $g s c$-null mice were predominantly restricted to derivatives of the first branchial arch, which correlate with the later pattern of $g s c$ expression. In addition, the rib cage abnormalities also correlate with the expression in the developing ventrolateral body wall. One significant region of embryonic gsc expression where no defects were detected were the limbs. Perhaps, like gastrulation, gsc also has a redundant role in limb development.

Craniofacial development is a complex and dynamic process involving numerous tissue interactions (Noden, 1988). The expression of $g s c$ in cranial mesenchyme and the craniofacial defects observed in our $g s c$-null mice suggest that this homeoprotein is involved in inductive tissue interactions that form the head. Many of the craniofacial structures that were abnormal in the gsc mutants are derived from the neural crest that migrate into the cranial region. Previous studies have shown that gsc expression can modulate cell migration in Xenopus embryos (Niehrs et al., 1993). It is unlikely that $g s c$ is regulating neural crest cell migration because gsc expression in neural crest-derived cells occurs after migration (Hunt et al., 1991). Thus, gsc may be important for regulating postmigratory neural crest-derived cell behaviour in response to inductive signals that is essential for proper tissue morphogenesis. Two other homeobox genes, $m s x$ - 1 and Mhox, are expressed in cranial mesenchyme and, when mutated, also result in numerous craniofacial defects (Satokata and Maas, 1994; Martin et al., 1995). Some of the abnormalities found in the these mutant mice overlap with those of our $g s c$ mutants but most are different. Thus, multiple homeoproteins expressed in cranial mesenchyme function uniquely in the formation of the various components of the vertebrate head. It is also interesting to note that $g s c$-null mice have abnormalities in sensory organs that may alter olfaction and hearing. The involvement of cranial neural crest in the development of the sensory organs has been proposed to be fundamental to vertebrate evolution (Gans and Northcutt, 1983). Thus, gsc appears to play an important evolutionary role in vertebrate head morphogenesis.

We thank Jenny Deng for assistance with tissue culture, Hector Lujan for help with the analysis of the mutant mice, Allan Bradley for the AB-1 ES and SNL 76/7 STO cell lines and the C57BL/6-albino mice, Hiroshi Sasaki and Brigid Hogan for the HNF3 $\beta$ antibody and Liz Robertson for critical reading of the manuscript. This work was supported by a National Institutes of Health grant HD31155 and a grant from the Sid W. Richardson Foundation to R. R. B.

\section{REFERENCES}

Ang, S.-L., Wierda, A.,Wong, D., Stevens, K. A., Cascio, S., Rossant, J. and Zaret, K. S. (1993). The formation and maintenance of the definitive endoderm lineage in the mouse: involvement of HNF3/forkhead proteins. Development 119, 1301-1315.

Ang, S.-L. and Rossant, J. (1994). HNF-3 $\beta$ is essential for node and notochord formation in mouse development. Cell 78, 561-574.

Beddington, R. S. P. (1994). Induction of a second neural axis by the mouse node. Development 120, 613-620.

Blum, M., Gaunt, S. J., Cho, K. W. Y., Steinbeisser, H., Blumberg, B., Bittner, D. A. and De Robertis, E. M. (1992). Gastrulation in the mouse: The role of the homeobox gene goosecoid. Cell 69, 1097-1106.
Blumberg, B., Wright, C. V. E., De Robertis, E. M. and Cho, K. W. Y. (1991). Organizer-specific homeobox genes in Xenopus laevis embryos. Science 253, 194-196.

Bradley, A. (1987). Production and analysis of chimeric mice. In Teratocarcinomas and Embryonic Stem Cells: A Practical Approach (ed. E. J. Robertson). pp. 113-151. Oxford: IRL Press.

Cho, K. W. Y., Blumberg, B., Steinbeisser, H. and De Robertis, E. M. (1991). Molecular nature of Spemann's organizer: the role of the Xenopus homeobox gene goosecoid. Cell 67, 1111-1120.

De Robertis, E. M., Blum, M., Niehrs, C. and Steinbeisser, H. (1992). goosecoid and the organizer. Development 1992 Supplement, 167-171.

Gans, C. and Northcutt, R. G. (1983). Neural crest and the origin of vertebrates: a new head. Science 220, 268-274.

Gaunt, S. J., Blum, M. and De Robertis, E. M. (1993). Expression of the mouse goosecoid gene during mid-embryogenesis may mark mesenchymal cell lineages in the developing head, limbs and body wall.Development 117, 769-778.

Green, J. B. A., New, H. V and Smith, J. C. (1992). Responses of embryonic Xenopus cells to activin and FGF are separated by multiple dose thresholds and correspond to distinct axes of the mesoderm. Cell 71, 731-739.

Hunt, P., Wilkinson, D. and Krumlauf, R. (1991). Patterning the vertebrate head: murine Hox 2 genes mark distinct subpopulations of premigratory and migrating cranial neural crest. Development 112, 43-50.

Izpisúa-Belmonte, J. C., De Robertis, E. M., Storey, K. G. and Stern, C. D. (1993). The homeobox gene goosecoid and the origin of organizer cells in the early chick blastoderm. Cell 74, 645-659.

Jegalian, B. G. and De Robertis, E. M. (1992). Homeotic transformations in the mouse induced by overexpression of a human Hox3.3 transgene. Cell 71, 901-910.

Kochhar, D. M. (1973). Limb development in mouse embryos. I. Analysis of teratogenic effects of retinoic acid. Teratology 7, 289-298.

Mansour, S. L., Thomas, K. R. and Capecchi, M. R.(1988). Disruption of the proto-oncogene int- 2 in mouse embryo-derived stem cells: a general strategy for targeting mutations to non-selectable genes. Nature 336, 348-352.

Martin, J. F., Bradley, A. and Olson, E. N. (1995). The paired-like homeo box gene MHox is required for early events of skeletogenesis in multiple lineages. Genes Dev. 9, 1237-1249.

McMahon, A. P. and Bradley, A. (1990). The Wnt-1 (int-1) proto-oncogene is required for development of a large region of the mouse brain. Cell 62, 10731085.

Niehrs, C., Keller, R., Cho, K. W. Y. and De Robertis, E. M. (1993). The homeobox gene goosecoid controls cell migration in Xenopus embryos. Cell 72, 491-503.

Noden, D. M. (1988). Interactions and fates of avian craniofacial mesenchyme. Development 104 Supplement, 121-140.

Sasaki, H. and Hogan, B. L. M. (1993). Differential expression of multiple fork head related genes during gastrulation and axial pattern formation in the mouse embryo. Development 118, 47-59.

Satokata, I. and Maas, R. (1994). Msxl deficient mice exhibit cleft palate and abnormalities of craniofacial and tooth development. Nature Genetics $\mathbf{6}$, 348-355.

Schulte-Merker, S., Hammerschmidt, M., Beuchle, D., Cho, K. W. Y., De Robertis, E. M. and Nüsslein-Volhard, C. (1994). Expression of zebrafish goosecoid and no tail gene products in wild-type and mutant no tail embryos. Development 120, 843-852.

Shawlot, W. and Behringer, R. R. (1995). Requirement for Liml in headorganizer function. Nature $\mathbf{3 7 4}, 425-430$.

Soriano, P., Montgomery, C., Geske, R. and Bradley, A. (1991). Targeted disruption of the $c$-src proto-oncogene leads to osteopetrosis in mice. Cell 64, 693-702.

Spemann, H. and Mangold, H. (1924). Überinduktion von embryonanlagen durch implantation artfremder organisatoren. Wilhelm Roux's Arch. Dev. Biol. 100, 599-638.

Stachel, S. E., Grunwald, D. J. and Myers, P. Z. (1993). Lithium perturbation and goosecoid expression identify a dorsal specification pathway in the pregastrula zebrafish. Development 117, 1261-1274.

Steinbeisser, H., De Robertis, E. M., Ku, M., Kessler, D. S. and Melton, D. A. (1993). Xenopus axis formation: induction of goosecoid by injected Xwnt8 and activin mRNAs. Development 118, 499-507.

Weinstein, D. C., Ruiz i Altaba, A., Chen, W. S., Hoodless, P., Prezioso, V. R., Jessell, T. M. and Darnell, J. E., Jr. (1994). The winged-helix transcription factor $H N F-3 \beta$ is required for notochord development in the mouse embryo. Cell 78, 575-588.

(Accepted 30 May 1995) 